\title{
Synthèse des journées scientifiques francophones portant sur les codes de calculs en radioprotection, radiophysique et dosimétrie
}

\author{
D. PAUL ${ }^{1}$, L. MAKOVICKA ${ }^{2}$, M. RICARD ${ }^{3}$
}

(Manuscrit reçu le 9 juillet 2004, accepté le 27 décembre 2004)

RÉSUMÉ L'article dresse le bilan des études présentées lors des journées scientifiques francophones co-organisées le 2-3 octobre 2003 à Sochaux par la SFRP (Société française de radioprotection), la SFPM (Société française de physique médicale) et la FIRAM (Association francophone pour le développement et l'utilisation des logiciels de simulation d'interaction rayonnement matière). L'article s'attache tout particulièrement à restituer de ces journées internationales, la synthèse des tendances actuelles dans le domaine du développement et de l'utilisation des codes de calculs en radioprotection, en radiophysique et en dosimétrie.

ABSTRACT Synthesis of the scientific French speaking days on numerical codes in radiation protection, in radiophysics and in dosimetry.

The paper carries the title of "French speaking" scientific days co-organized on October 2-3, 2003 in Sochaux by the SFRP, SFPM and FIRAM societies. It has for objective to establish the scientific balance sheet of this international event, to give the synthesis of current tendencies in the field of the development and of the use of the numerical codes in radiation protection, in radiophysics and in dosimetry.

Key words: Numerical codes / Monte Carlo Simulation / Radiation Protection / Radiophysics / Dosimetry

\section{Introduction}

Le développement des codes de calculs utilisant des méthodes analytiques et celles de type « Monte Carlo » est spectaculaire et étroitement lié aux progrès nouvelles performances des moyens informatiques. Les codes sont devenus également plus accessibles et plus conviviaux, facilitant ainsi leur implantation et leur exploitation au sein non seulement des laboratoires de recherche mais aussi des industries, des sociétés de service ou des hôpitaux. L'utilisateur n'est plus nécessairement un

\footnotetext{
1 Service de Médecine et Santé au travail, Laboratoire de Biogénotoxicologie et Mutagenèse Environnementale (EA 1784IFR PMSE 112), Université de la Méditerranée, Faculté de Pharmacie, 27 boulevard Jean Roulin, 13385 Marseille Cedex 05, France.

2 IRMA/CREST/FEMTO-ST, UMR 6174 du CNRS, Pôle universitaire, B.P. 71427, 25211 Montbéliard Cedex, France.

3 Service de Physique, Institut Gustave Roussy, 39 rue Camille Desmoulins, 94805 Villejuif Cedex, France.
} 


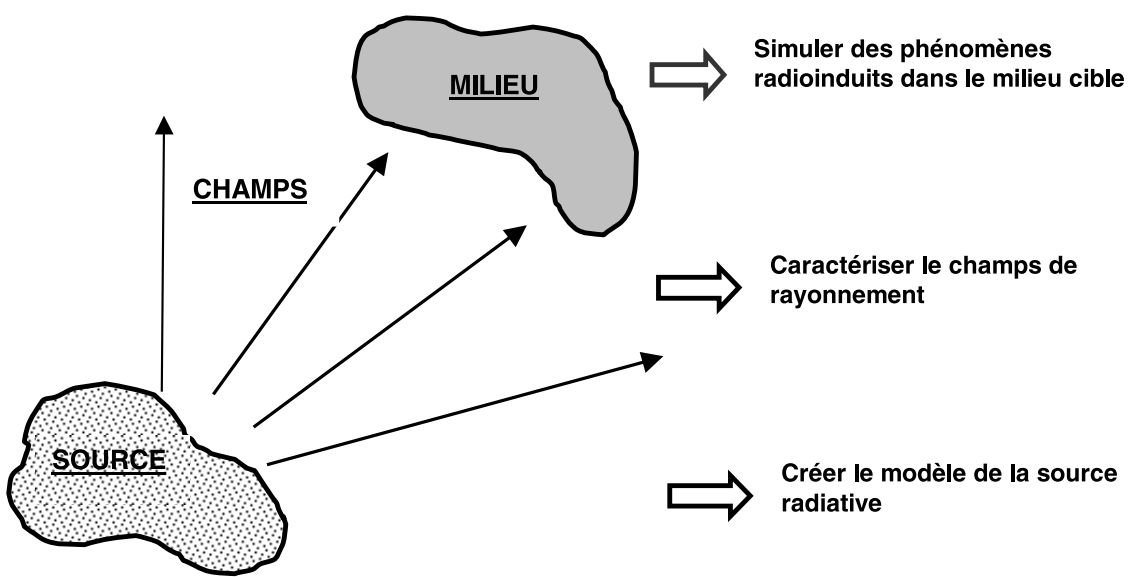

Figure 1 - Le problème de la simulation représenté généralement en trois étapes successives. The problem of the simulation generally represented in three successive steps.

développeur soucieux d'appliquer son propre code, un chercheur théoricien, mais un scientifique faisant face à un problème, dont la solution échappe en partie à l'expérience. Les utilisateurs étant nombreux et variés aujourd'hui, les organisateurs ont tenté naturellement d'associer des partenaires représentatifs, pour faire un état de l'art des codes de calcul de transport des rayonnements, d'estimation de sources et de gestion de doses.

L'élargissement thématique des journées SFRP a probablement contribué, en comparaison avec les journées de 1996 (SFRP, 1996) à l'augmentation de la variété des applications dans le domaine médical, de la métrologie ou du développement de l'instrumentation et de nouvelles méthodes. Il serait, néanmoins, subjectif et prématuré de dresser un bilan complet et exhaustif, basé uniquement sur les présentations des 2-3 octobre 2003, des avancées et de l'utilisation dans les pays francophones des codes de simulation.

Le plan de l'article suit les caractéristiques et évolutions des différents codes utilisés dans les domaines suivants : la radioprotection industrielle et la radioprotection des installations lourdes, l'instrumentation et la métrologie, les applications médicales.

\section{Caractéristiques et évolutions des codes}

Même si le problème des simulations peut, la plupart du temps, être résumé par son schéma original (Fig. 1), la spécificité de certaines applications conduit à une spécialisation des codes et assez souvent à leur utilisation croisée, soit directe avec 
la création de codes combinés, soit indirecte sous forme d'inter comparaisons ou de validations systématiques par d'autres codes.

Le schéma (Fig. 1) conduit obligatoirement, et indépendamment du domaine d'applications, à la spécialisation des codes. On peut toujours grossièrement distinguer des codes de trois types : les codes d'estimation des sources, les codes de transport et les codes de gestion de dose, mais la tendance générale évolue vers le regroupement des codes «multifonctions ».

L'ensemble des communications présentées apporte la preuve logique que l'approche du problème et les méthodes utilisées divergent d'un domaine d'application à l'autre au niveau des codes d'estimation des sources. Si les codes de transport permettent aujourd'hui de mieux approcher la recherche industrielle, instrumentale et médicale, ce sont surtout les codes de gestion de doses qui représentent le vrai facteur commun et donnent la meilleure possibilité de communication et d'échange entre les différentes disciplines.

Les codes « maison », assez fréquents encore dans les années 90, et traitant de manière générale les aspects physiques de bases mais avec les caractéristiques de conception optimisées en fonction d'un problème spécifique, deviennent de plus en plus rares (Nerio et al., 2003 ; Rottner, 2003) et n'apparaissent la plupart du temps que sous la forme de simples sous programmes des codes plus importants. Les « grands codes Monte Carlo généralistes » de type MCNP (Monte Carlo N Particles), GEANT (Detector Description and Simulation Tool), PENELOPE (PENetration and Energy Loss of Positrons and Electrons), TRIPOLI (Energy dependent three dimensional Monte Carlo code), EGS (Electron Gamma Shower), entre autres grâce à leur évolution quasi permanente, dominent le marché et se trouvent fréquemment intégrés au cœur de codes nouveaux. L'activité de la banque de données nucléaires de l'Agence pour l'énergie nucléaire (AEN) (Kodeli et Sartori, 2003) contribue à ce phénomène et le facilite par le principe de son fonctionnement. Elle assure la distribution d'environ 4000 programmes de calcul (Fig. 2), de bibliothèques de données nucléaires et d'expériences intégrales par an. Elle représente au sein de l'Organisation de coopération et de développement économique (OCDE) un moteur de développement de la recherche dans les domaines concernés, elle permet également d'orienter les utilisateurs en organisant des séminaires ou des cours de formation sur des programmes de «référence». Elle travaille également en étroite collaboration avec l'Agence internationale de l'énergie atomique (AIEA) de Vienne, avec la Commission européenne et elle assure les échanges avec le Canada et les États-Unies. 


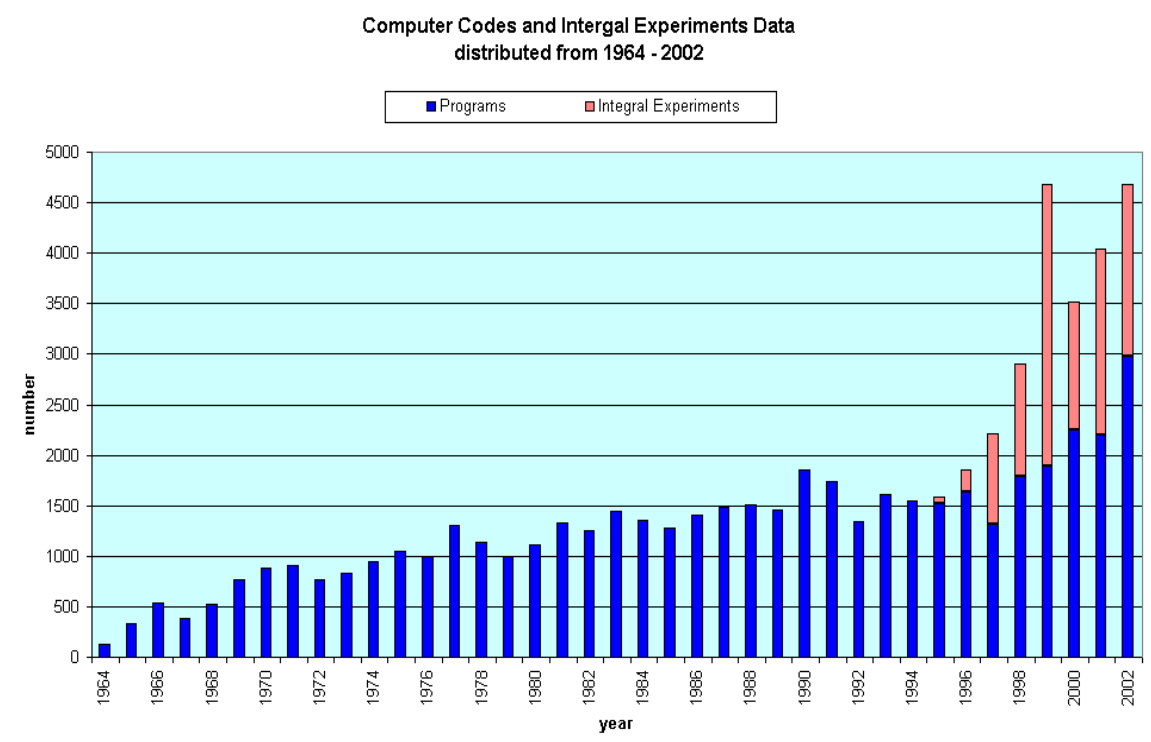

Figure 2 - Évolution de la distribution des codes de calculs distribués par l'AEN (Kodeli et Sartori, 2003) entre 1964 et 2002 : programmes et expériences.

Evolution of the codes distributed from 1964-2002 by NEA (Kodeli and Sartori, 2003): programs and experiments.

\subsection{La radioprotection industrielle et la radioprotection des installations lourdes}

La majorité des codes utilisés est basée sur les concepts des versions antérieures. Les versions nouvelles comme par exemple TRIPOLI-4 (SFRP, 1996 ; Both et al., 2003) n'apportent, dans la plupart des cas, sur le plan de la physique, que des améliorations partielles et les changements les plus significatifs se situent plutôt sur le plan de nouvelles méthodologies informatiques et de l'exploitation plus large des bibliothèques de données. Un certain nombre de caractéristiques fondamentales nouvelles a été cependant incorporé (sections efficaces ponctuelles, géométrie combinatoire, calcul de perturbations). TRIPOLI-4, code «français », est utilisé principalement pour quatre domaines d'application: les études de protection, les études de criticité, les études de cœurs des réacteurs et les études d'instrumentation. Il continue à faire partie de la famille des «grands» codes de référence, fiable, avec un champ d'application vaste et une convivialité croissante. 
Le code MERCURE (3D-code industriel de propagation des gamma par la méthode d'atténuation en ligne droite) (Suteau et al., 2003) a évolué depuis les journées organisées en 1996 vers la version 6.3. Rappelons-nous que ce code simule le transport de photons de $15 \mathrm{keV}$ à $10 \mathrm{MeV}$ dans les géométries 3D entre des sources volumiques et des points de calcul. Il permet de déterminer aussi bien le débit d'équivalent de dose que le Kerma dans l'air ou le flux énergétique. La méthode utilisée est celle de l'intégration de noyaux ponctuels d'atténuation en ligne droite avec facteurs d'accumulation. À noter qu'il existe une version industrielle de ce code, MERCURAD (3D-Simulation Software for dose rate calculation), munie d'une interface graphique homme machine, permettant de construire des scènes complètes. Le code MERCURE a été comparé au code TWODANT (Two-Dimensional, Diffusion-Accelerated, Neutral Particles Transport, Los Alamos) à des fins de validation.

La fonctionnalité et l'utilisation du formulaire DARWIN (formulaire) développé dans le cadre de programmes R\&D ont été illustrées (Tsizanizara et al., 2003) par différentes applications pratiques : calculs de puissance résiduelle d'un combustible, établissement d'une cartographie complète d'activité dans le cas du démantèlement d'une centrale nucléaire, activation d'une cible... Le formulaire continue à subir des améliorations afin de satisfaire les besoins en qualité exigés par les utilisateurs industriels ou les chercheurs.

Les codes spécifiques développés pour des situations ou applications bien précises comme CECRAC (Codes d'évaluation des conséquences radiologiques d'un accident de criticité) (Beaujard et al., 2003), PANTHERE (Prévisions et analyses théoriques de l'exposition dans les réacteurs) (Guiges et Tribollet, 2003) ou l'évaluation des doses autour du système hybride MYRRHA (A multipurpose accelerator driven system for research and development) (Aoust, 2003) gardent leur importance dans le paysage de la radioprotection industrielle (Fig. 3) mais s'adressent logiquement à un nombre d'utilisateurs relativement limité.

À l'opposé des codes précédemment mentionnés, les différentes versions du code MCNP permettent de couvrir pratiquement tous les secteurs de la radioprotection. Le domaine de la radioprotection industrielle n'y fait pas exception, car ce code sert souvent pour les comparaisons ou les validations des codes plus spécifiques (Aoust, 2003) et des applications variées de la radioprotection (Borne et Choplin, 2003 ; Antoni et al., 2003 ; Verboomen et al., 2003). Une attention particulière est donnée dans la plupart de ces codes à la description matérielle et géométrique des sites étudiés. À titre d'exemple des résultats tout à fait satisfaisants ont été obtenus dans le cadre des travaux menés au SCK-CEN avec l'usage combiné du scanner gamma et de l'outil de planification VISIPLAN 3D (3D ALARA planning tool for nuclear environment) (Vermeersch, 


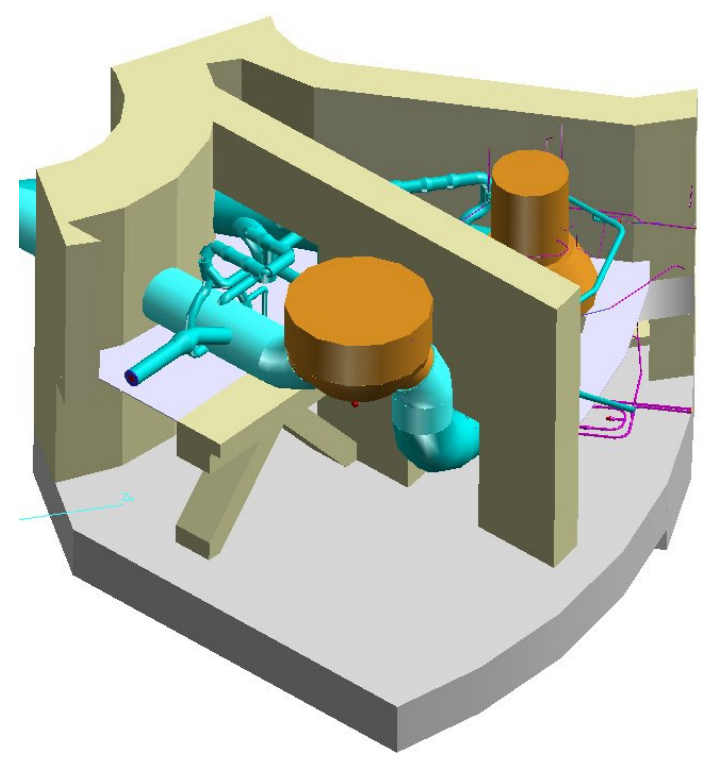

Figure 3 - La modélisation d'une géométrie complexe dans PAN. THERE-Pallier 900 Mwe Boucle $3 \mathrm{GV}+$ pompe 4 (Guiges et Tribollet, 2003).

The modelling of a complex geometry in PANTHER, 900 MWe Buckle $3 G \mathrm{~V}+$ pump 4 (Guiges and Tribollet, 2003).
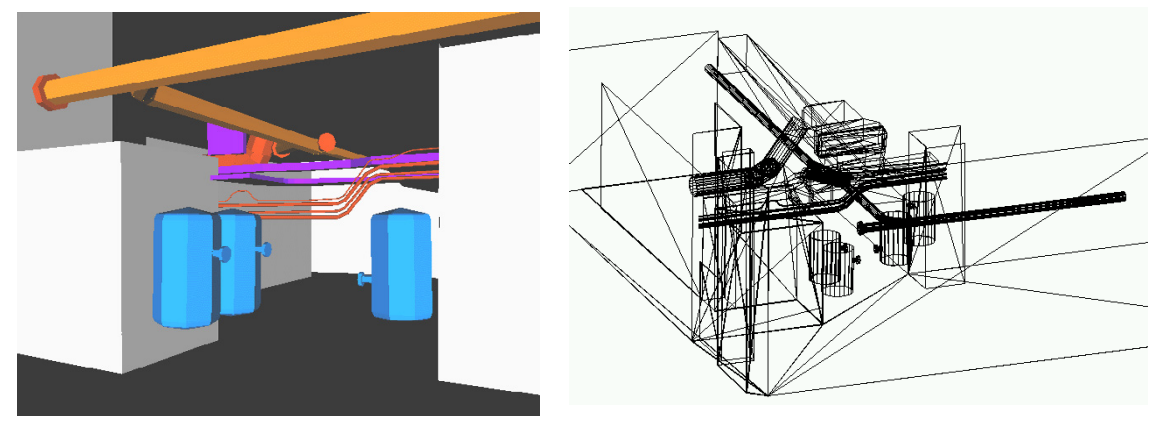

Figure 4 - Modèle cartographié et modèle VISIPLAN de la géométrie du site (Vermeersch, 2003). Model cartography and VISIPLAN model of the site geometry (Vermeersch, 2003).

2003). La méthode présentée permet d'établir des modèles radiologiques adéquats du poste du travail (Fig. 4). Ce logiciel de planification calcule les doses justement à partir des données relatives à la géométrie, aux matériaux et aux sources en se basant sur la méthode du « point-kernel » avec la correction de «build-up ».

On peut constater que depuis 1996 l'évolution de ce secteur d'utilisation de codes n'a pas apporté des changements radicaux mais poursuit son chemin en optimisant, en améliorant la précision des résultats obtenus, en s'orientant souvent 
vers l'utilisation combinée de différents codes et/ou de leur biaisage (Quéinnec et Ménard, 2003).

\subsection{L' instrumentation et la métrologie}

Ces domaines d'applications des codes de calculs, essentiellement ceux basés sur la méthode de Monte Carlo, se montrent être le «point de rencontre » ou une sorte de passerelle entre le monde industriel et le monde médical. Les problèmes liés par exemple à la dosimétrie rencontrent souvent les mêmes difficultés, à une échelle comparable et avec des exigences sur la précision souhaitée assez proches. Les principaux codes utilisés sont aussi souvent les mêmes.

Les codes de calculs permettent en outre la détermination de grandeurs souvent inaccessibles par l'expérience et même si leur utilisation a pris un réel essor grâce au progrès de l'informatique certaines zones d'ombre liées à l'incertitude inhérente aux sections efficaces persistent à ce jour. Les simulations numériques, surtout à basses énergies, ne permettent pas d'atteindre la précision absolue requise en métrologie et les calculs n'apportent que des coefficients de correction applicables aux données expérimentales. L'évaluation de l'erreur systématique, en dehors de l'erreur statistique, pose également dans certaines applications de nombreuses difficultés qui influent sur la qualité attendue des prédictions obtenues par simulation.

Le bilan des codes généralistes utilisés par la plupart des équipes peut être basé sur celui fait au CEA/LNHB (Laboratoire National Henri Becquerel, Barthe et al., 2003).

Le code de Monte Carlo EGS4 (Nelson et al., 1985) est l'un des premiers codes mis à la disposition des chercheurs pour simuler le transport du couple électron/photon dans la matière. Il a été utilisé et éprouvé avec succès dans un grand nombre d'utilisations en métrologie des rayonnements. Néanmoins, dans certaines situations, comme la mesure dans une chambre d'ionisations, le code ne délivre pas de résultats suffisamment fiables. Une version plus récente, connue sous le nom de EGSnrc, répond partiellement à ces problèmes. Notons également que la source écrite en langage MORTRAN, basé sur l'utilisation de macroinstructions, doit être systématiquement compilée en Fortran. L'avantage de cette approche est l'obtention d'un code plus compact et plus facile à manipuler pour l'utilisateur.

Le code MCNP4 (Breismeister, 2000) «suit » les particules neutres (photons et neutrons), les électrons (positifs et négatifs) et est étendu à certaines particules lourdes chargées dans sa version MCNPX. Ce code est directement lié à l'origine 
au projet Manhattan. Initialement, ce code ne prenait en compte que le transport des particules non chargées comme les neutrons et photons. MCNP fait l'objet d'un développement continu depuis les années quarante soutenu par une politique d'assurance qualité. Au-delà de la modélisation de l'interaction du rayonnement avec la matière, il dispose d'outils statistiques pour déterminer la convergence mathématique du résultat obtenu et de techniques de réduction de variance intégrées. Il ne nécessite pas une re-compilation du code pour chaque cas à traiter comme dans EGS.

Le code GEANT 4.0 développé au sein du Conseil européen pour la recherche nucléaire (CERN) se présente sous la forme d'une bibliothèque de données et d'outils que l'utilisateur peut assembler ensemble selon ses besoins spécifiques (object-oriented technology). La dernière version est écrite en langage $\mathrm{C}++$ au lieu du Fortran habituellement utilisé par les autres codes évoqués dans cet article. Il est très complet en ce qui concerne les types de particules qui sont suivies : photons, électrons, hadrons et ions.

PENELOPE (Salvat et al., 2001) a été conçu à l'université de Barcelone dans le but de suivre dans un premier temps uniquement les électrons et les positrons. Le transport des photons fut ajouté par la suite. L'un des avantages de ce code sur les précédents est sa réalisation récente qui incorpore un certain nombre de concepts physiques plus modernes et plus précis avec une unité de conception plus grande et rigoureuse. Comme la plupart de ce type de code, il est écrit en Fortran 77.

Le grand avantage des codes généralistes est leur grande souplesse d'adaptation aux caractéristiques géométriques et matérielles du problème à résoudre. Par contre, dans certains cas, ils présentent une faiblesse relative au fait qu'ils ont été initialement conçus pour des particules de hautes énergies que l'on trouve auprès des grands accélérateurs comme ceux du Stanford Linear Accelerator Center (code EGS4), du CERN (code GEANT 3.0). Cette faiblesse de conception se trouve amplifiée par la méconnaissance des sections efficaces d'interaction aux basses énergies évoquée précédemment.

Certaines faiblesses de codes généralistes dans les domaines énergétiques extrêmes peuvent être comblées par l'élaboration de codes spécifiques couplés aux précédents ; exemple, le couplage du code CPAT (Centre de physique des plasmas et de leurs applications de Toulouse) avec le code PENELOPE (Mitev et Segur, 2003).

Les inter-comparaisons obtenues pour les systèmes géométriques simples conduisent en règle générale à un bon accord (Fig. 5). La validation de résultats 
a)

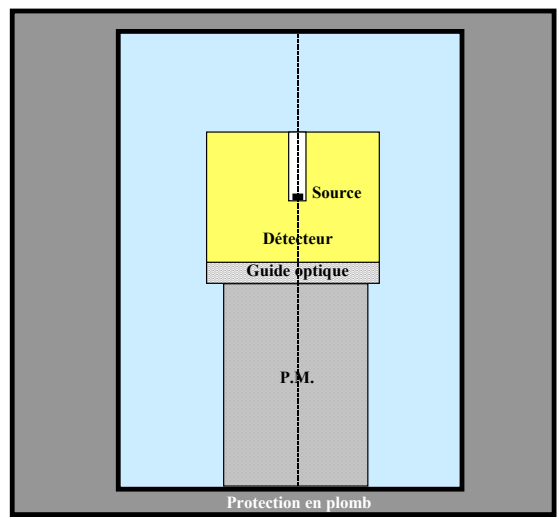

b)

Efficacité du détecteur puit du LNHB

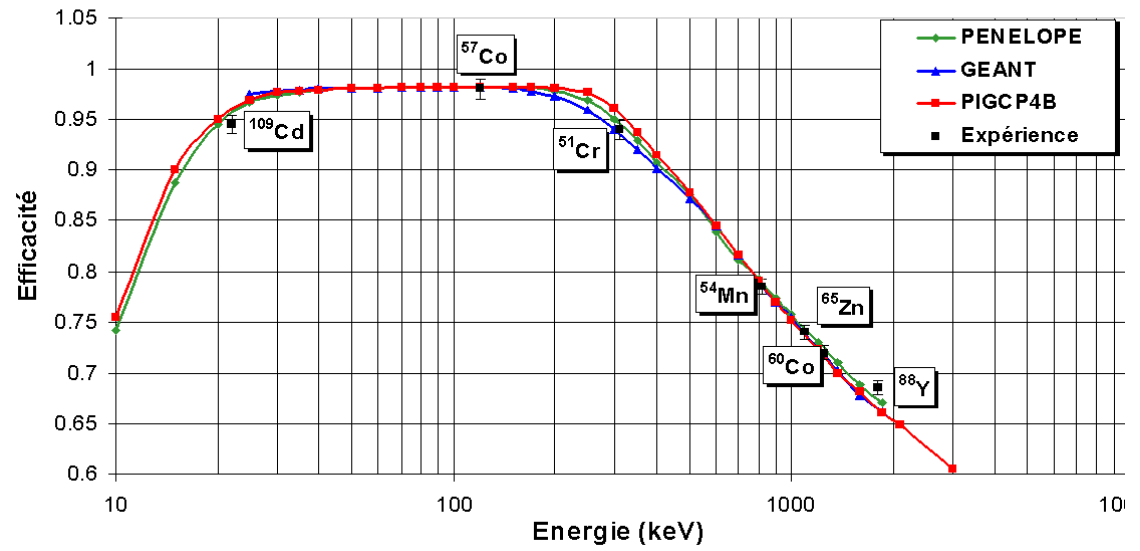

Figure 5 - (a) Schéma de principe du détecteur puit à scintillateur (Barthe et al., 2003). (b) Comparaison entre calculs et mesures pour le détecteur puit du LNHB.

(a) Principal plan of the well detector (Barthe et al., 2003). (b) Comparison between calculations and measurements for the LNHB detector.

par codes différents se montre utile dans les cas complexes ou dans les situations difficilement accessibles par la voie expérimentale.

Néanmoins le type des particules suivies joue un rôle non négligeable. La comparaison se montre en règle générale, notamment dans les applications industrielles (Aoust, 2003 ; Ménard, 2003 ; Lahaye, 2003), beaucoup plus délicate pour les neutrons ou les champs mixtes (Figs. 6a et 6b). Les simulations 
a)

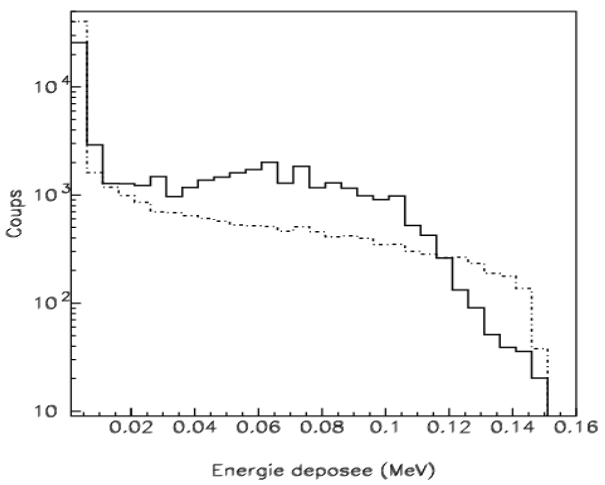

b)

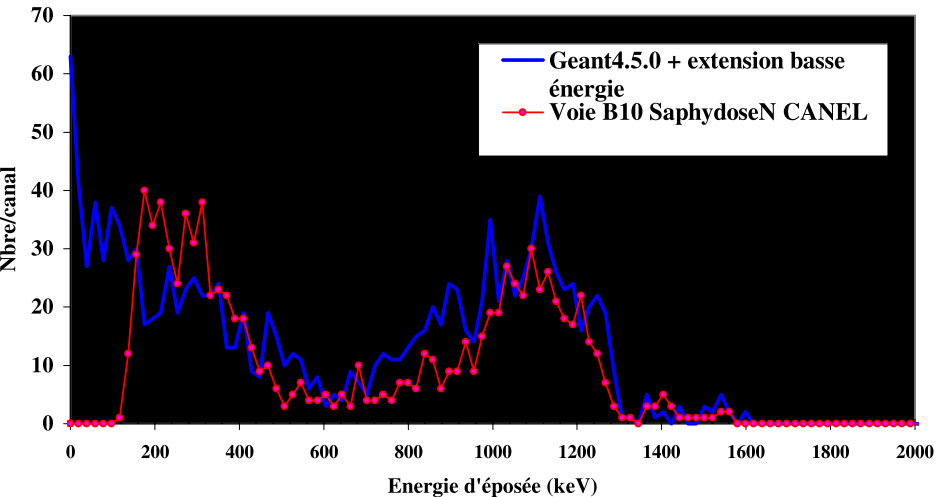

Figure 6 - (a) Spectres de dépôts d'énergie du compteur équivalent tissus cylindrique pour des neutrons de $565 \mathrm{keV}$ et des photons de $478 \mathrm{keV}$ (résultats expérimentaux en pointillés, numériques en continu) (Ménard, 2003). (b) Superposition du spectre calculé au spectre expérimental correspondant à une irradiation avec des neutrons thermiques (Lahaye, 2003).

(a) Spectra of energy deposition in the equivalent tissues cylindrical detector for neutrons of $565 \mathrm{keV}$ and photons of $478 \mathrm{keV}$ (experimental results: dotted line, calculations: in continuous) (Ménard, 2003). (b) Calculated and experimental results superimposed corresponding to exposure with thermal neutrons (Lahaye, 2003).

numériques ont dans ces cas pour objectif de donner une première approche ou une faisabilité évitant la multiplication des expériences (réduction de fabrication des prototypes) et permettant ainsi de réduire les coûts ou les délais.

L'utilisation de codes numériques pour la reconstitution dosimétrique d'accident radiologique et le développement de fantômes voxelisés s'apparente à celui de la « métrologie et l'instrumentation » en radiophysique et radioprotection médicale (Lemosquet et al., 2003 ; Pierrat et al., 2003). 


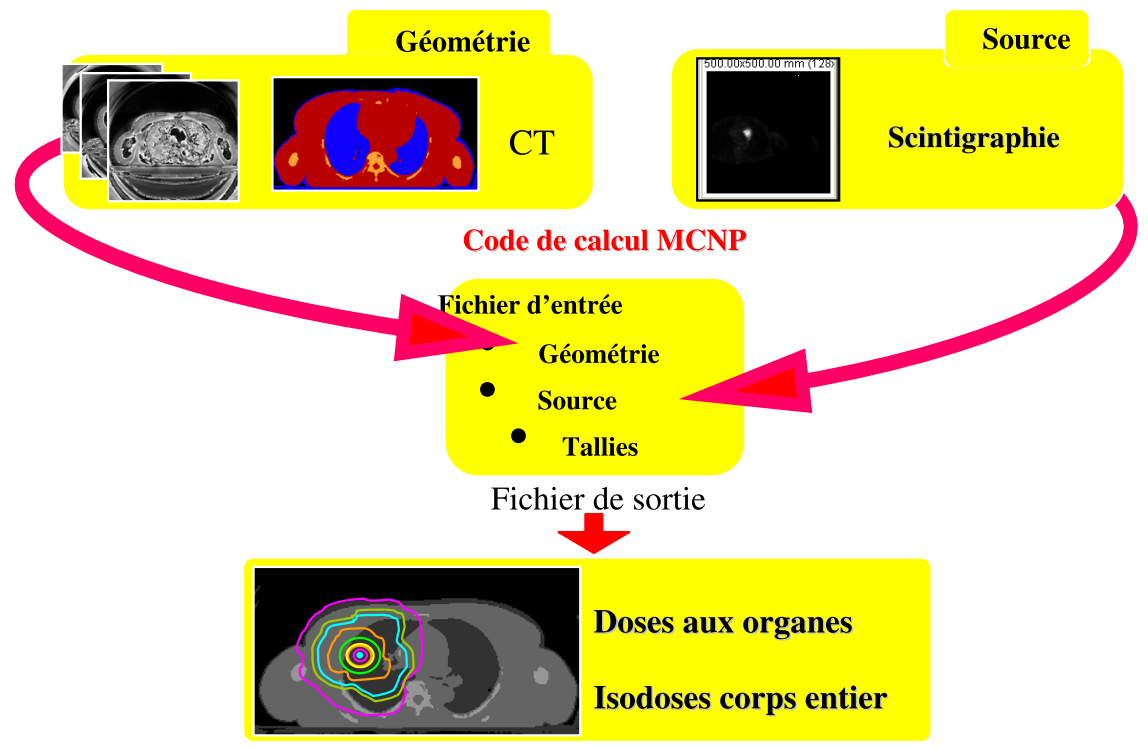

Figure 7 - Structure de l'outil de dosimétrie personnalisée constitué de l'interface graphique CEDIPE et du code MCNP (Chiavassa et al., 2003).

Structure of the dosimetric personal tool elaborated with the graphic interface CEDIPE and the MCNP code (Chiavassa et al., 2003).

\subsection{Applications médicales}

L'utilisation des codes de calcul dans le domaine médicale a plusieurs facettes :

- la radioprotection du personnel, du patient et l'optimisation instrumentale ;

- la radiothérapie externe ;

- la dosimétrie interne.

La radioprotection du personnel, du patient et l'optimisation instrumentale ne s'éloignent pas des problèmes précédemment exposés dans le cadre de la radioprotection générale dans les installations utilisant toutes sortes de rayonnements ionisants. Les techniques utilisées sont facilement transposables entre les différents domaines car les objectifs à atteindre restent très proches.

Les méthodes numériques utilisées sont à nouveau essentiellement des méthodes de Monte Carlo, très souvent basées sur une extension ou amélioration des grands codes généralistes préexistants : EGS-BEAM, GEANT-GATE (Geant Application for Tomographic Emission), EEDIPE (Outil d'Évaluation de la dose interne personnalisée), MCNP... (Fig. 7). La polyvalence du code MCNP se 
confirme par son exploitation (Bourhis-Martin et al., 2003) dans le cas de traitements radiothérapeutiques spécifiques comme la thérapie par capture de neutrons (BNCT).

Les objectifs constants des chercheurs en radiobiologie, radiothérapie et radioprotection restent, d'une part, l'établissement d'un rapport entre événements physiques initiaux et effets biologiques observés avec une approche globalisante (macroscopique) et d'autre part, ceux liés au calcul de trace qui suit les interactions des particules mises en jeu jusqu'au bout de leur trajet pour modéliser les effets initiaux des rayonnements avec un regard plutôt microscopique à l'échelle de l'acide désoxyribo-nucléique (ADN) (Terrissol, 2003; Martin, 2003). Cette approche «microscopique » contribue efficacement au développement des techniques thérapeutiques spécifiques ou combinées comme la PAT (Photo Activation Therapy). L'originalité et l'importance d'un tel modèle de calcul simulant la photoactivation, l'émission Auger, la fluorescence X et le transport de toutes ces particules dans un nucléosome (core particle) et permettant l'évaluation des dommages primaires à l'ADN, sont indiscutables. Les simulations Monte-Carlo de tous ces événements physiques et chimiques, allant de la création des particules jusqu'à environ $10^{-8}$ seconde ont pu être effectuées et les rendements de cassures de brins d'ADN ont été déduits dans ce modèle en utilisant les ionisations directes de l'ADN et les réactions des espèces créées lors de la radiolyse de l'eau.

Les simulations effectuées en radiothérapie externe (Nahum, 2003; Gschwind et al., 2003 ; Guillerminet, 2003) confirment les énormes avantages des techniques Monte Carlo pour la planification des traitements et pour une meilleure connaissance des sources dépassant souvent très largement les informations fournies par les constructeurs. Le fait que les techniques conformationnelles utilisées en radiothérapie externe : radiothérapie stéréotaxique, IMRT (Intensity Modulated Radiation Therapy), fenêtrage... exigent d'obtenir des distributions complexes de la dose afin de cibler au mieux le volume tumoral à traiter en protégeant le mieux possible les tissus sains avoisinant le volume cible, met en valeur la puissance et la souplesse des modèles numériques. Le progrès informatique des deux dernières décennies a rendu les simulations Monte Carlo tout à fait abordables dans le cas des particules chargées (électrons) (Fig. 8) mais ces dernières restent, malgré des efforts considérables fournis dans le cadre de l'accélération des calculs (techniques de la réduction de variance), assez délicates pour être utilisées en routine de manière systématique dans le cas des irradiations photoniques. La réduction des 3 étapes intermédiaires conduisant vers un modèle complet (Fig. 9) permet de rendre grâce à l'exploitation de «l'espace des phases » des simulations aussi plus efficaces. 


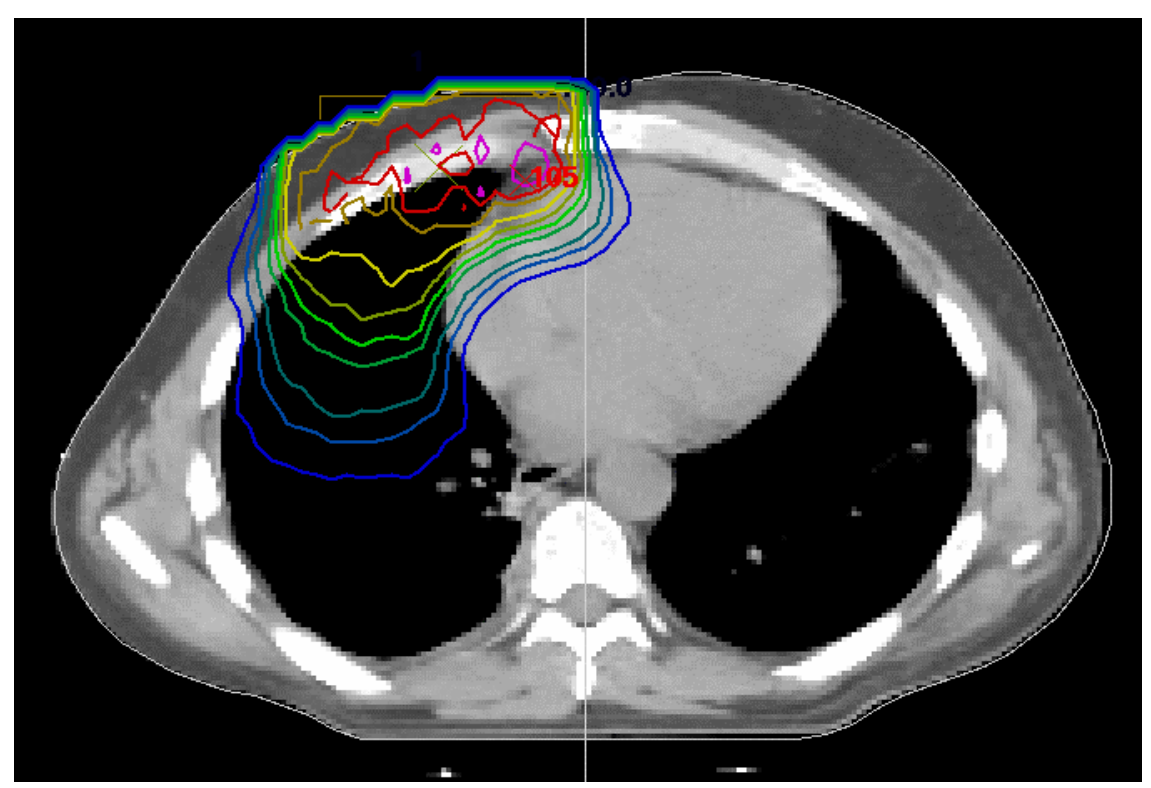

Figure 8-Planification des traitements de poumons par faisceau d'électrons (16 MeV; champ $10 \times$ $10 \mathrm{~cm}^{2}$ ) avec les isodoses calculées par EGS4/BEAM (Nahum, 2003).

Electron-beam treatment plan in the lungs with the dose distribution shown as isodose lines (16 MeV beam; $10 \times 10 \mathrm{~cm}^{2}$ field) calculated with EGS4/BEAM (Nahum, 2003).

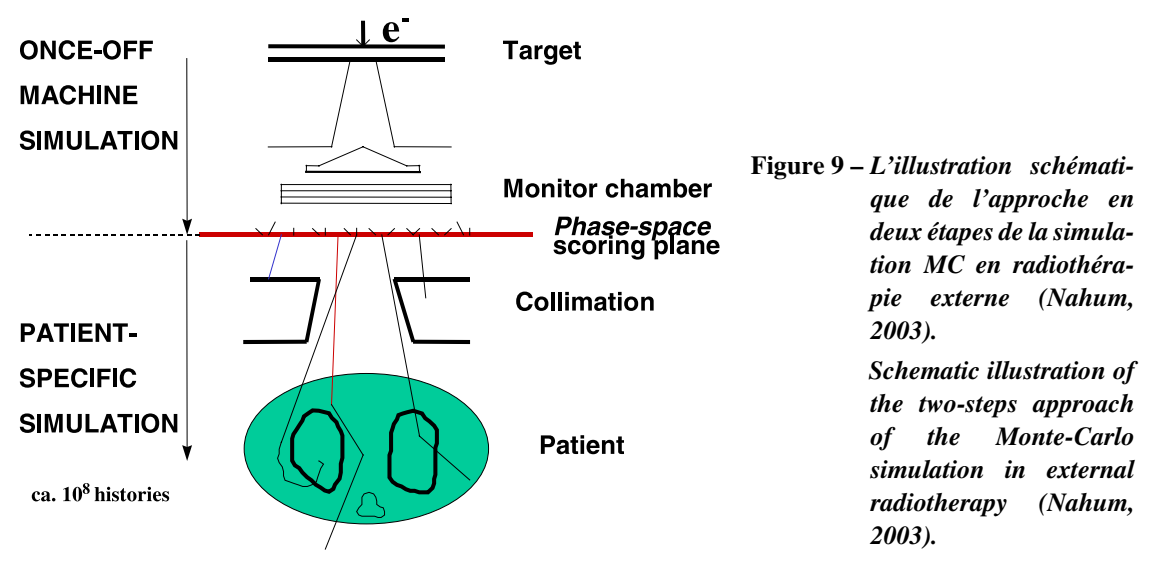


Une nouvelle voie qui pourrait considérablement améliorer les temps de calculs en radiothérapie externe en gardant la souplesse et la précision des méthodes de Monte Carlo, semble être l'utilisation des réseaux de neurones artificiels (RNA) mais ces études ne sont qu'à leurs débuts (Mathieu et al., 2003).

L'amélioration des performances des moyens de calculs actuels est souvent très rapidement stimulée par les exigences croissantes au niveau de la description précise 3D des fantômes. Cette problématique joue également un rôle important en dosimétrie interne et en radioprotection de manière très générale (Ricard et Coulot, 2003 ; Coulot et al., 2003 ; Pierrat et al., 2003 ; Chiavassa et al., 2003).

\section{Conclusion}

Les travaux présentés au cours des Journées SFRP confirment la tendance générale qui fait apparaître clairement durant la dernière décennie, d'utilisation très dominante de méthodes de Monte Carlo par rapport aux autres techniques de simulation dans les domaines liés à la radiophysique.

Il peut sembler à première vue qu'il y a parmi les utilisateurs des codes de calculs, deux classes distincts : d'un côté, les industriels (recherche appliquée, exploitation d'installation nucléaire, instrumentation) ; d'un autre côté, le monde médical (recherche médicale, laboratoires).

Les problématiques peuvent apparaître actuellement assez différentes car d'une part l'industrie (ou ce qui peut être classé comme recherche industrielle) est en règle générale confrontée aux problèmes stationnaires, d'assez grands volumes et de fortes atténuations nécessitant des techniques de biaisage, d'autre part le milieu médical cherche à résoudre des problèmes très hétérogènes, avec de courtes distances, conduisant plutôt à l'usage unique des codes de simulation (sans biaisage) mettant l'accent sur la précision dosimétrique. L'explication de ce décalage réside probablement dans la priorité des objectifs d'un secteur à l'autre. Les aspects physiques, dosimétriques et métrologiques représentent clairement une sorte de « passerelle » entre les deux mondes. On peut d'un côté considérer que les utilisateurs «industriels » de codes, probablement moins représentatifs dans l'ensemble, travaillant sur l'amélioration des performances de l'appareillage de détection sont souvent confrontés aux mêmes problématiques que les utilisateurs «médicaux» ciblant essentiellement le bilan ou l'efficacité des traitements, d'un autre côté leurs collègues, utilisateurs des codes dédiés à la radioprotection du personnel et des patients, certes minoritaires à ce jour, rencontrent obligatoirement des problèmes très proches de la radioprotection dite « industrielle ». 


\section{RÉFÉRENCES}

Les références signalées par [*] font parties d'actes des Journées scientifiques francophones « Codes de calculs en radioprotection, radiophysique et dosimétrie », SFRP-SFPM-FIRAM, le 2-3 octobre 2003, Sochaux, consultables sur le site http://www.sfrp.asso.fr.

Antoni R., Bourgois L., Comte N. (2003) Applications des codes de calculs de radioprotection au SPR Saclay [*].

Aoust T. (2003) Évaluation de dose pour le MYRRHA ADS design - Utilisation MCNPX [*].

Barthe J., Gouriou J., Daures J., Ostrowsky A., Bordy J.M. (2003) Utilisation de codes de Monte Carlo en métrologie des rayonnements ionisants [*].

Beaujard P., Biaut G., Doursout T. (2003) CECRAC (code d'évaluation des conséquences radiologiques d'un accident de criticité) [*].

Borne F., Choplin P. (2003) Expertise dosimétrique auprès d'une installation Laser de puissanceUtilisation du code Monte Carlo MCNPX [*].

Both J.P., Mazzolo A., Peneliau Y., Petit O., Roesslinger B., Lee Y.K. (2003) La propagation des neutrons, des gamma, des électrons et des positrons dans la matière par la méthode de Monte Carlo : le code de transport 3D TRIPOLI-4 [*].

Bourhis-Martin E., Wojnecki C., Moss R., Antal G., Kiger W.S., Wittig A., Sauerwein W. (2003) Application du code de calcul MCNP à la thérapie par capture de neutrons - BNCT [*].

Breismeister J.F. (2000) MCNP, A General Monte Carlo N-particle Transport Code - Version 4C', Manuel LA-13709-M, 10 Avril 2000.

Chiavassa S., Aubineau-Laniece I., Bardies M., Martin S. (2003) Dosimétrie personnalisée en radioimmunothérapie à l'aide de fantômes voxélisés [*].

Coulot J., Faggiano A., Schlumberger M., Lavielle F., Aubert B., Ricard M. (2003) Étude de l'influence de l'hétérogénéité de distribution du radionucléide en dosimétrie interne par méthode de Monte Carlo [*].

Gschwind R., Guillerminet C., Makovicka L. (2003) Utilisation du code BEAM en radiothérapie externe [*].

Guigues L., Tribollet T. (2003) PANTHERE-Logiciel pour la prévision des débits de dose [*].

Guillerminet C. (2003) Utilisation de codes de type Monte Carlo pour déterminer la fluence en énergie des particules diffusées issues de l'accélérateur d'électrons à usage médical. Contribution à la dosimétrie 3D dans les milieux hétérogénes, thèse $n^{\circ} 76$, Université de Franche-Comté.

Kodeli I., Sartori E. (2003) Le rôle de la NEA Data Bank dans le domaine des codes de calcul en radioprotection, radiophysique et dosimétrie [*].

Lahaye T. (2003) Intérêt de la dosimétrie numérique en radioprotection : moyen de substitution ou consolidation des mesures [*].

Lemosquet A., Clairand I., De Carlan L., Aubineau-Laniece I., Bottollier-Depois J.F., Franck D. (2003) Application des fantômes voxels pour la reconstitution numérique d'accidents radiologiques [*].

Martin C. (2003) Modélisation des dommages radioinduits sur l'ADN : Prise en compte des radicaux libres et des réparations primaires, thèse, Université Paul Sabatier-Toulouse.

Mathieu R., Contassot-Vivier S., Guillerminet C., Gschwind R., Makovicka L., Bahi J. (2003) Prospective de la planification des traitements radiothérapeutiques basée sur les réseaux de neurones [*].

Menard S. (2003) Modélisation avec le code MCNPX de réponses sous rayonnements de dosimètres électroniques de R\&D commercialisés [*].

Mitev K., Segur P. (2003) Modélisation numérique du transport des électrons dans un compteur proportionnel cylindrique. Influence de la rétrodiffusion aux parois ; couplage du code CPAT3 avec le code PENELOPE [*]. 
Nahum A. (2003) Utilisation des codes de Monte Carlo pour la planification des traitements en radiothérapie externe [*].

Nelson W.R., Hirayama H., Rogers D.W.O. (1985) The EGS4 code system, Rapport SLAC-265, Standford Linear Accelerator Center, Standford, Californie.

Nerio R.U., Chica L.G., Paul D. (2003) Effet du rayon du cylindre d'argent d'une aiguille d'Iode-125, modèle 6711, sur la distribution du débit de dose autour de la source [*].

Pierrat N., Franck D., De Carlan L., Borissov N. (2003) Utilisation des fantômes voxels pour l'amélioration des étalonnages en anthroporadiamétrie [*].

Quéinnec F., Ménard S. (2003) Comparaison des efficacités de biaisage de Tripoli 4 et de MCNPX 2.4 pour un cas d'école [*].

Ricard M., Coulot J. (2003) Utilisation des codes de Monte Carlo en dosimétrie interne [*].

Rottner B. (2003) Code de calculs BRT_MC [*].

Salvat F., Fernandez-Varea J.M., Acosta E., Sempau J. (2001) PENELOPE - A code system for "Monte Carlo simulation of Electron and Photon transport", Rapport NEA/NSC/DOC(2001)19, ISBN 92-64-18475.

SFRP (1996) Actes Congrès SFRP, L'utilisation des codes de calculs en radioprotection, (http://www.sfrp.asso.fr), le 20-21 novembre 1996, INSTN Saclay.

Suteau C., Chiron M., Luneville L., Berger L., Huver M. (2003) MERCURE : un code 3D industriel de propagation des gamma par la méthode d'atténuation en ligne droite. Applications en radioprotection $[*]$.

Terrissol M. (2003) Modélisation des dommages induits après photoactivation d'atomes lourds incorporés à l'ADN [*].

Tsizanizara A., Huynh T.D., Luneville L., Diop C.M. (2003) Les fonctionnalités du code de calcul de la radioactivité DARWIN et les études de radioprotection [*].

Verboomen B., Coeck M., Baeten P. (2003) Évaluation du spectre des neutrons près du réacteur VENUS - Utilisation de MCNP [*].

Vermeersch F. (2003) Caractérisation et modélisation d'un site radioactif par usage combiné du scanner gamma unidirectionnel EDR et de l'outil de planification VISIPLAN 3D ALARA [*]. 